\title{
Synthesis of Chinensines A-E
}

\author{
Ioannis Margaros, Georgios Vassilikogiannakis*.
}

Department of Chemistry, University of Crete, Vasilika Vouton, 71003 Iraklion, Crete, Greece.

vasil@chemistry.uoc.gr
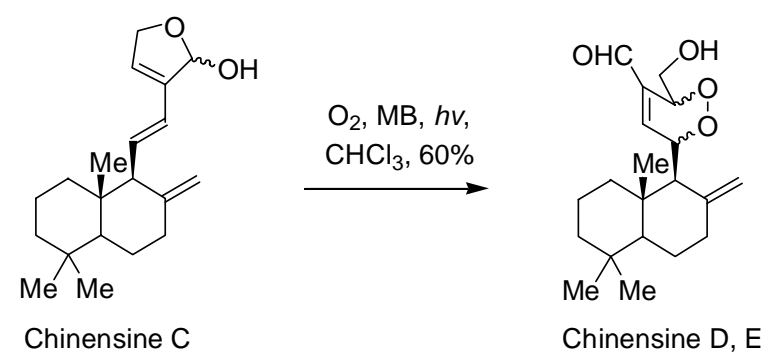

Supporting Information:

Page

Content

$\mathrm{S} 2$

General techniques

S3-S12

Copies of ${ }^{1} \mathrm{H}$ NMR and ${ }^{13} \mathrm{C}$ NMR 


\section{General Techniques:}

Reagents were purchased at the highest available commercial quality and used without further purification. All moisture sensitive reactions were carried out under a argon or nitrogen atmosphere. Anhydrous solvents were obtained as follows: THF distilled from sodium and benzophenone; $\mathrm{CH}_{2} \mathrm{Cl}_{2}$ distilled from $\mathrm{CaH}_{2}$; pyridine distilled from $\mathrm{KOH}$ pellets. Reactions were monitored by thin-layer chromatography (TLC) carried out on silica gel plates $\left(60 \mathrm{~F}_{254}\right)$ with UV light as the visualizing method and an acidic mixture of phosphomolybdic acid/cerium(IV) sulfate accompanied by heating of the plate as a developing system. The development agent contains $\mathrm{H}_{2} \mathrm{O}(94 \mathrm{~mL})$, concentrated $\mathrm{H}_{2} \mathrm{SO}_{4}$ $(6 \mathrm{~mL}), \mathrm{Ce}\left(\mathrm{SO}_{4}\right)_{2} \cdot\left(\mathrm{H}_{2} \mathrm{O}\right) \mathrm{n}(1.0 \mathrm{~g})$ and phosphomolybdic acid $(1.5 \mathrm{~g})$. Column chromatography was carried out on $\mathrm{SiO}_{2}$ (silica gel 60, particle size $0.040-0.063 \mathrm{~mm}$ ) with the specified eluent. ${ }^{1} \mathrm{H}$ and ${ }^{13} \mathrm{C}$ NMR spectra were recorded respectively at 500 $\mathrm{MHz}$ and $125 \mathrm{MHz}$, except ${ }^{13} \mathrm{C}$ NMR spectra of compounds $\mathbf{8}$ and $\mathbf{9}$, which were recorded at $75 \mathrm{MHz}$. All spectra are reported in $\delta(\mathrm{ppm})$ relative to the solvent residual peak (7.26 and $77.00 \mathrm{ppm}$ in $\mathrm{CDCl}_{3}$ ). The following abbreviations are used to explain the multiplicities: $\mathrm{s}=$ singlet, $\mathrm{d}=$ doublet, $\mathrm{t}=$ triplet, $\mathrm{q}=$ quartet, $\mathrm{m}=$ multiplet, $\mathrm{br}=\mathrm{broad}$. Irradiation experiments (photooxygenations) were performed with a Xenon $300 \mathrm{~W}$ lamp. Optical rotations were measured using a digital polarimeter (cell length $50 \mathrm{~mm}$ ). 


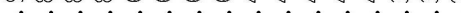

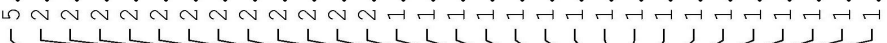<smiles>CC1(C)CCC[C@@]2(C)C1CC[C@@](C)(O)[C@H]2CC(=O)c1ccoc1</smiles>

11
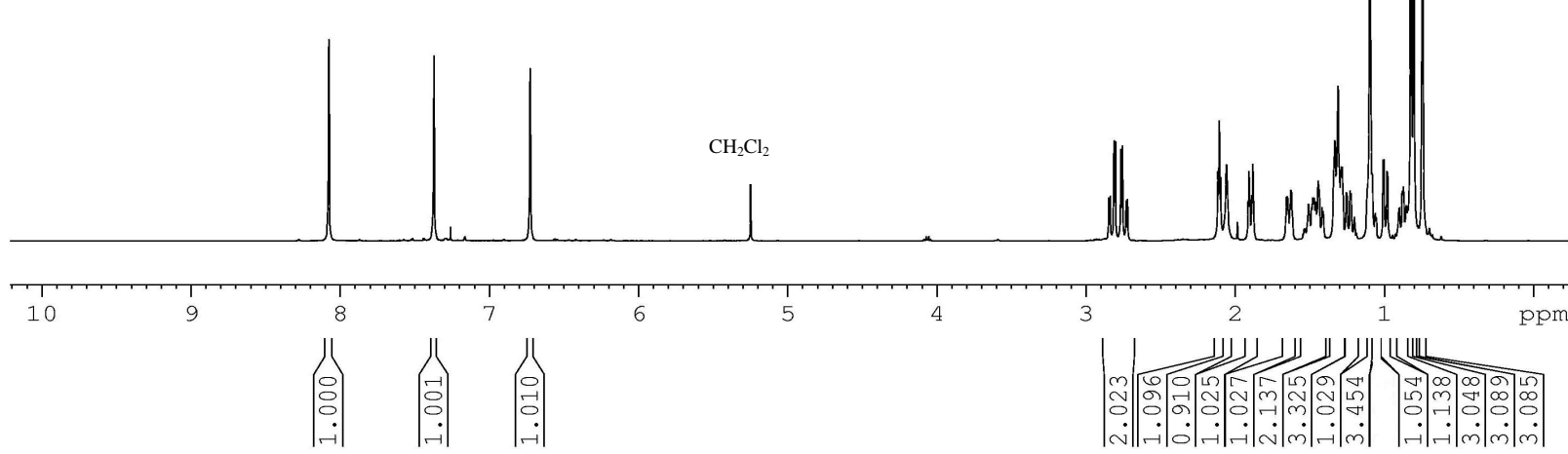

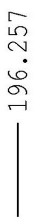
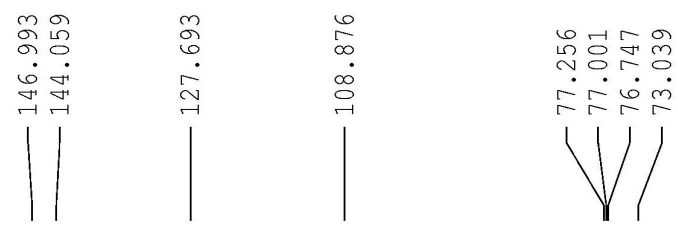

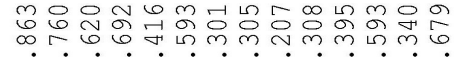

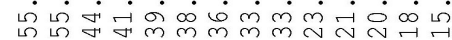<smiles>CC1(C)CCCC2(C)C(CC(=O)c3ccoc3)CCC12C</smiles>

11

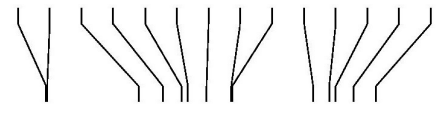

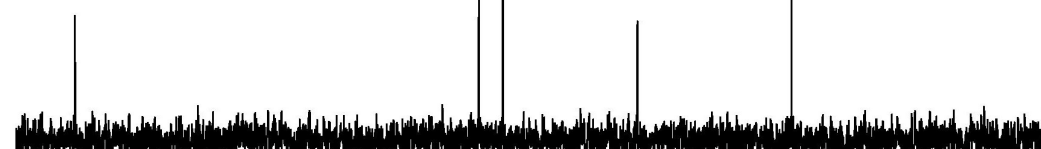

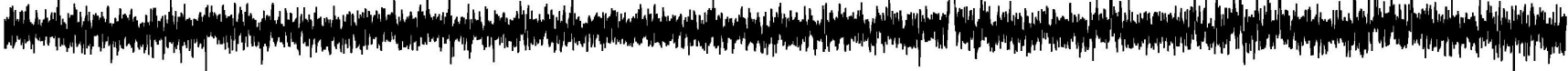

ता

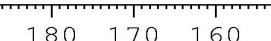

$150 \quad 140 \quad 130 \quad 120$

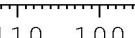

90

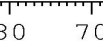

60 

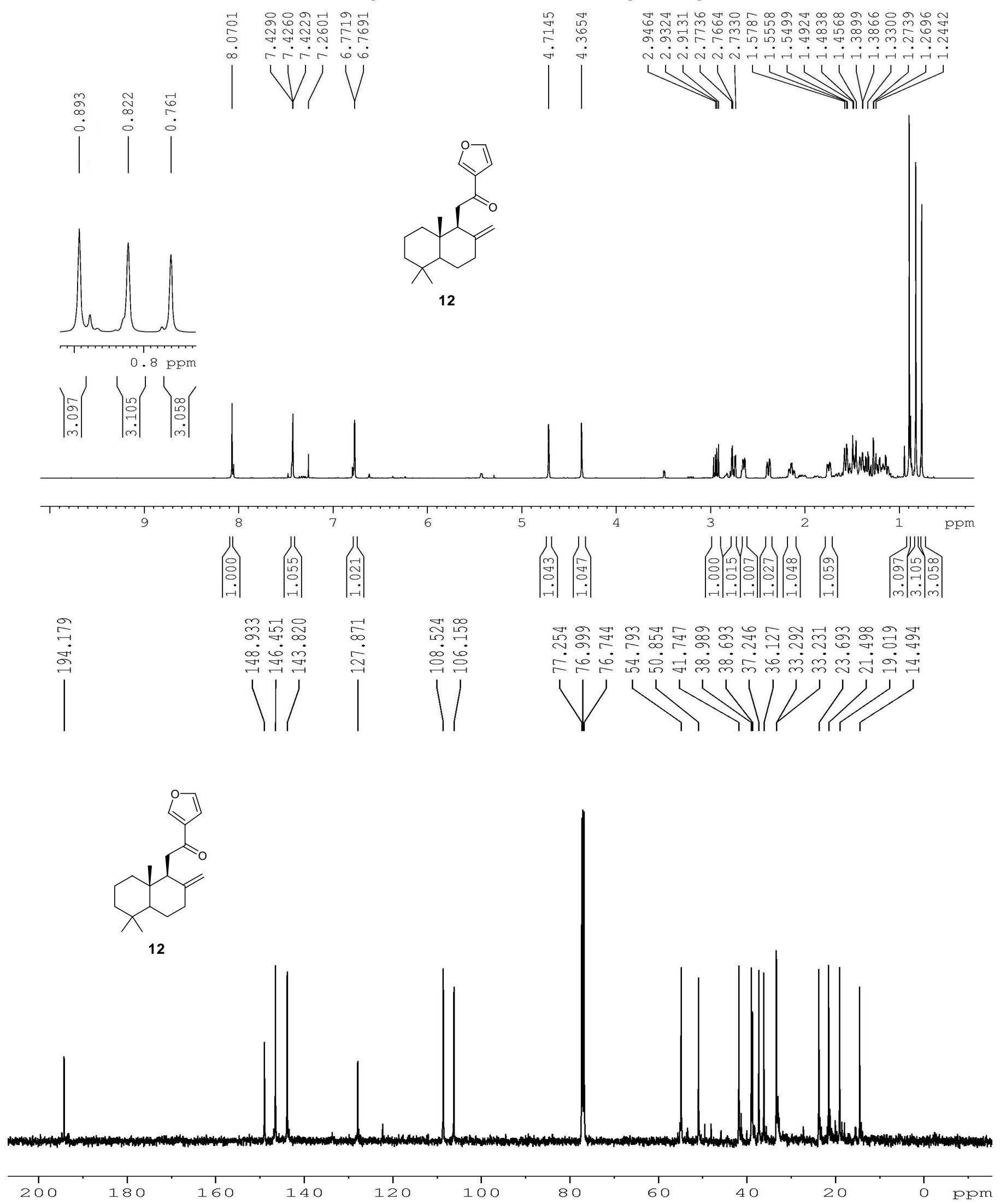

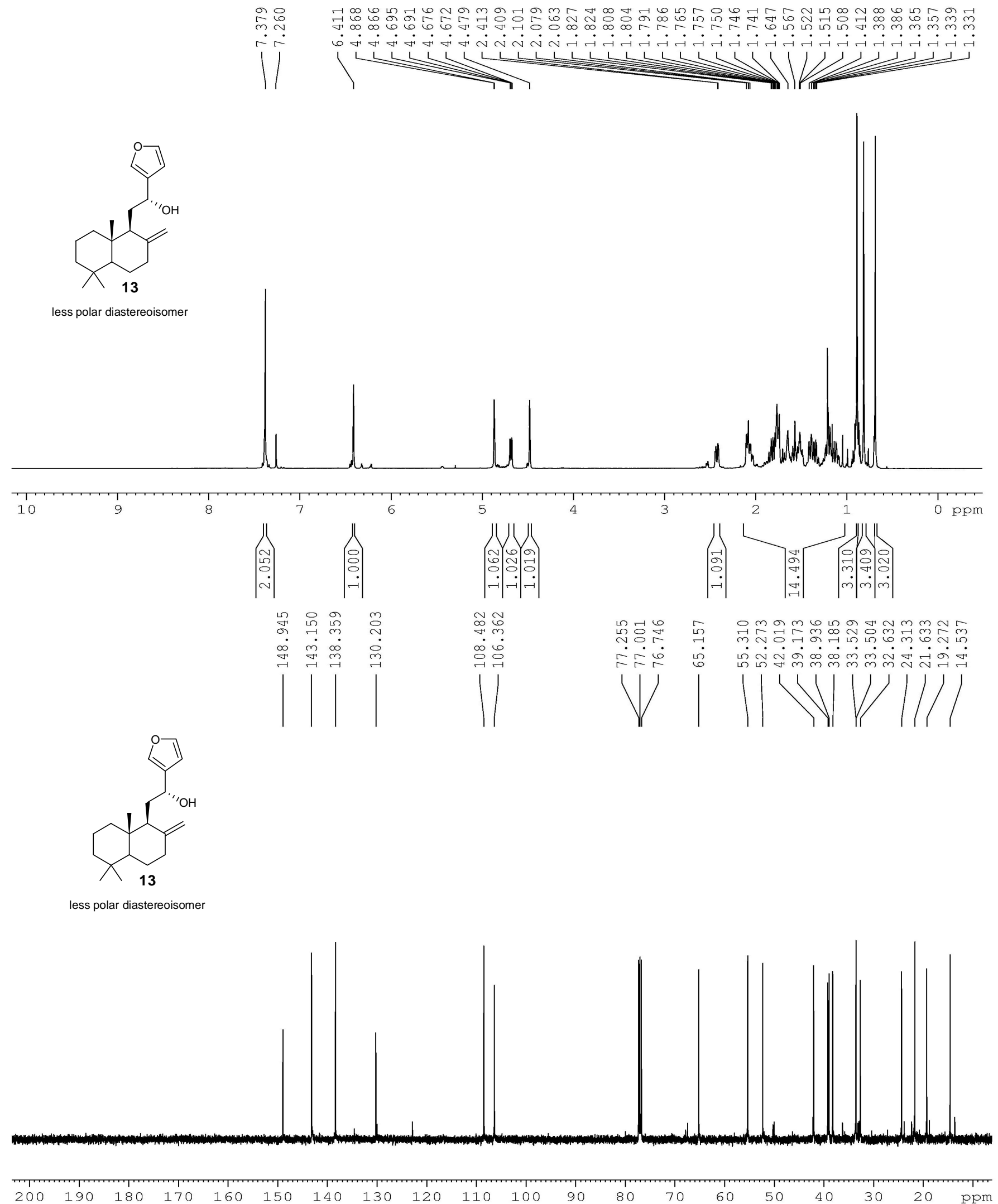

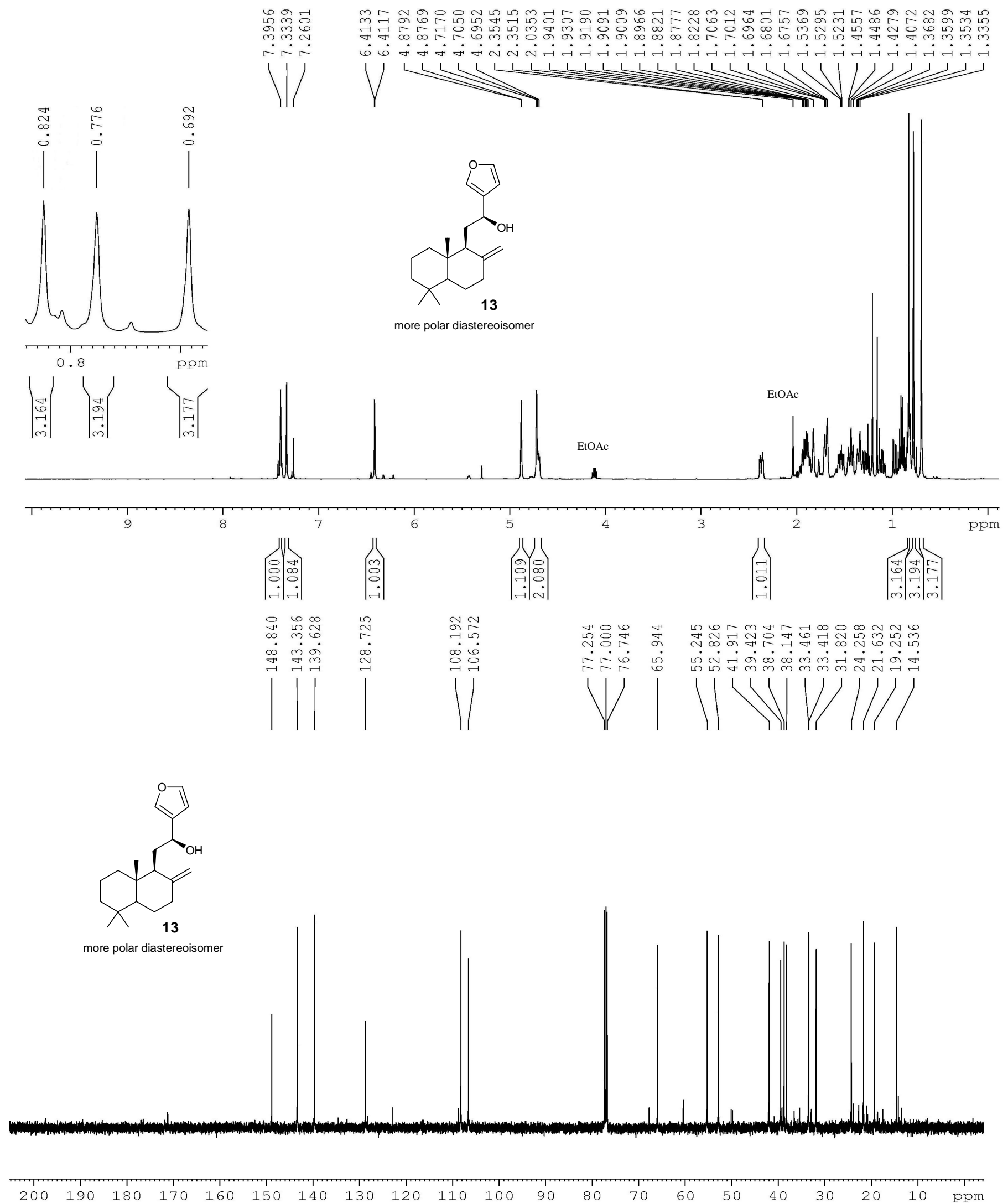

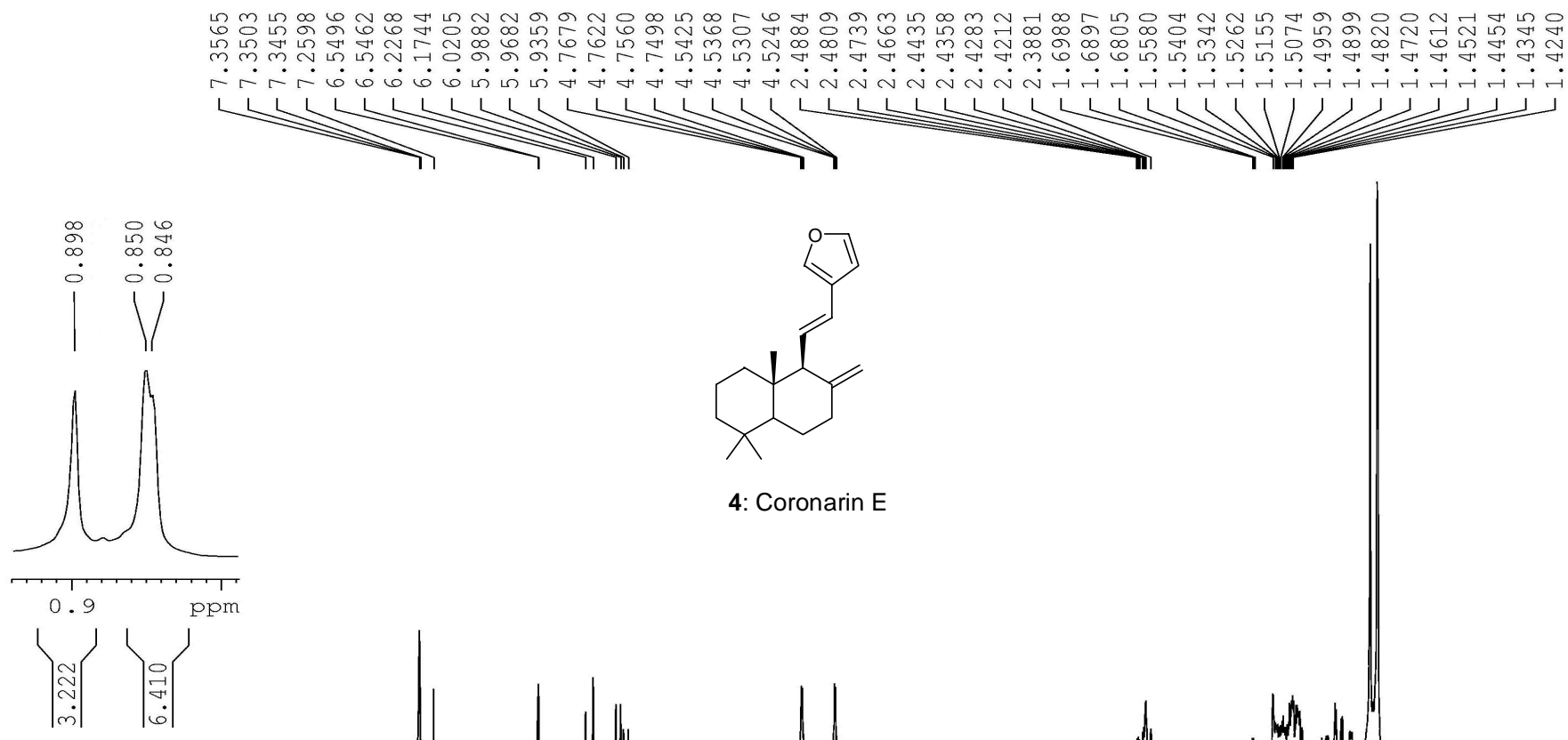

4: Coronarin E
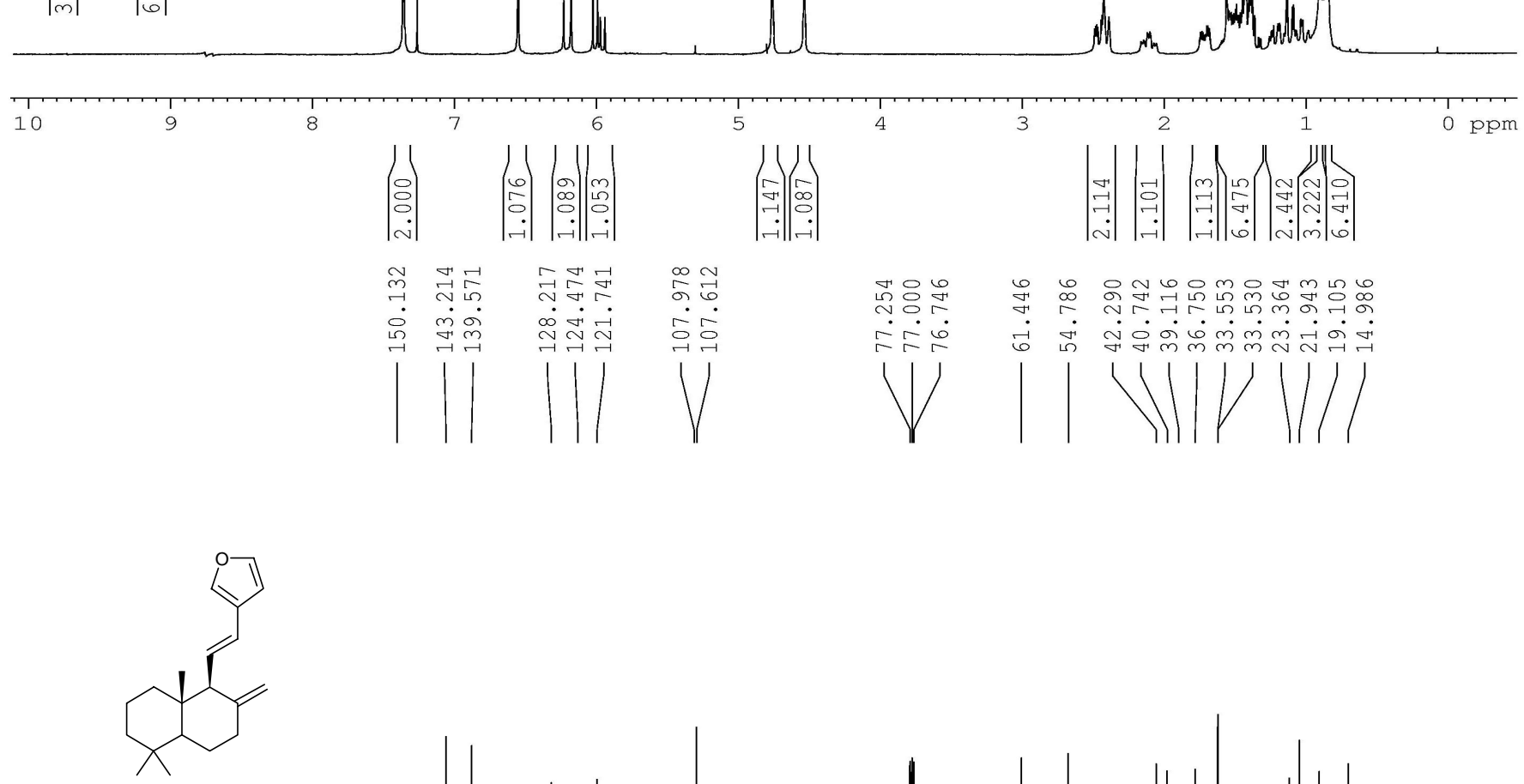

4: Coronarin E
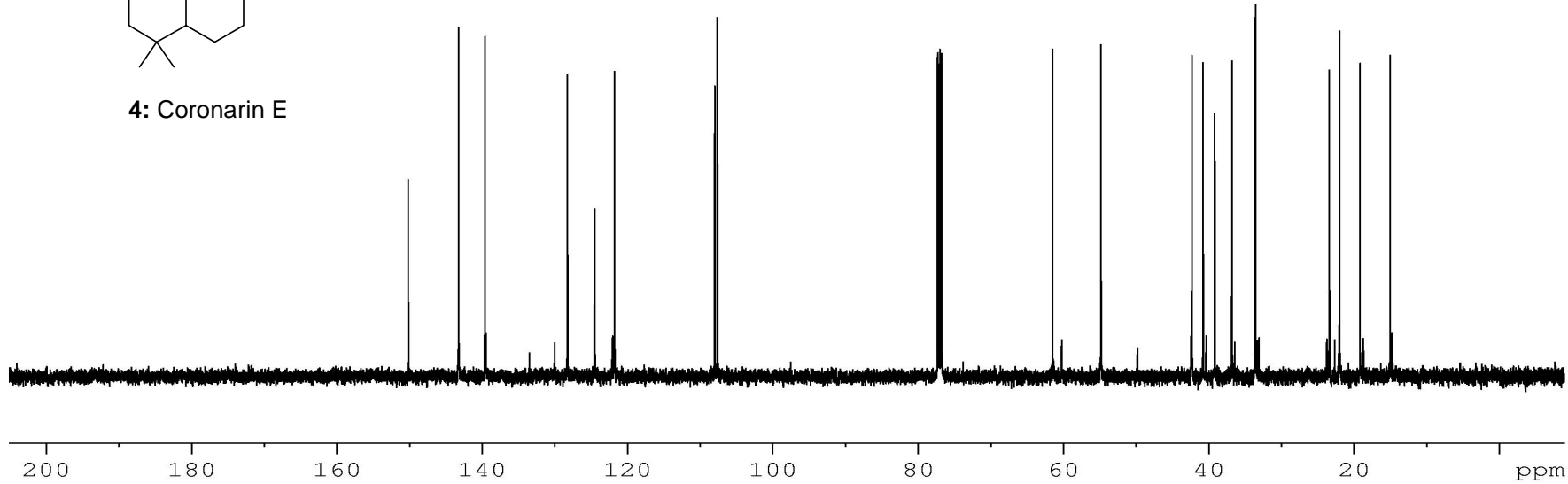


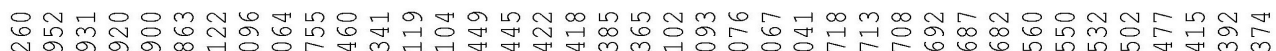

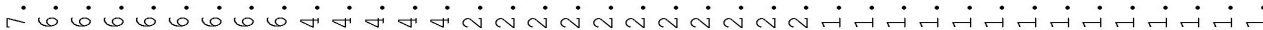

11111151
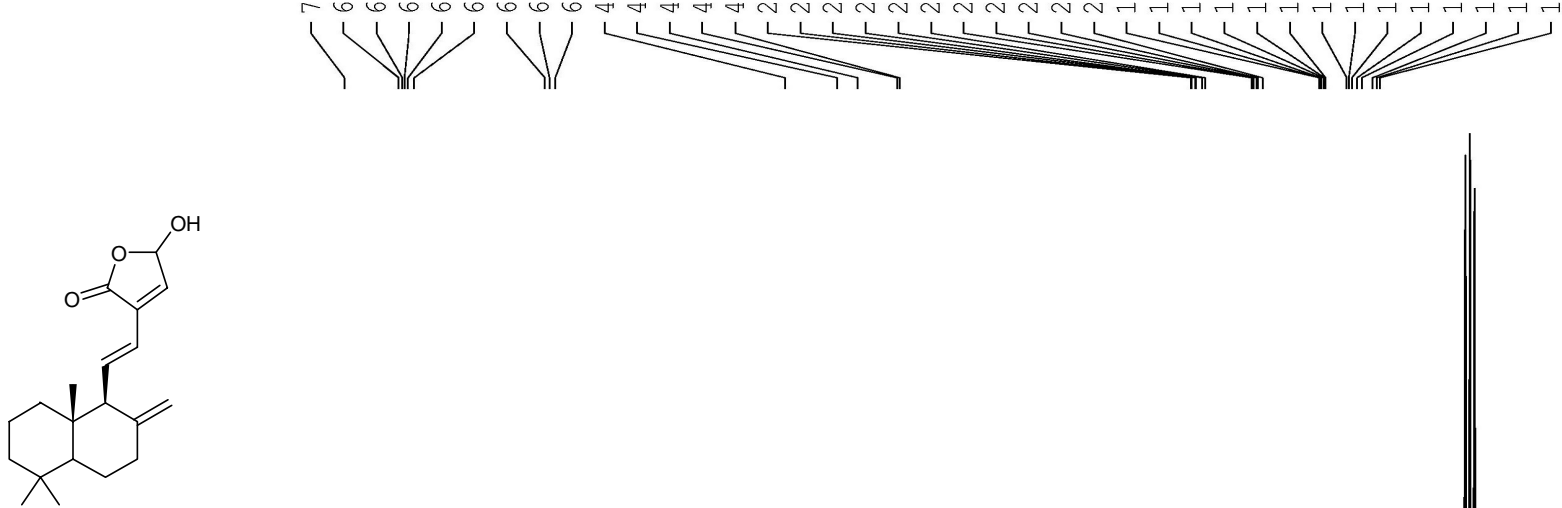

5: chinensine A

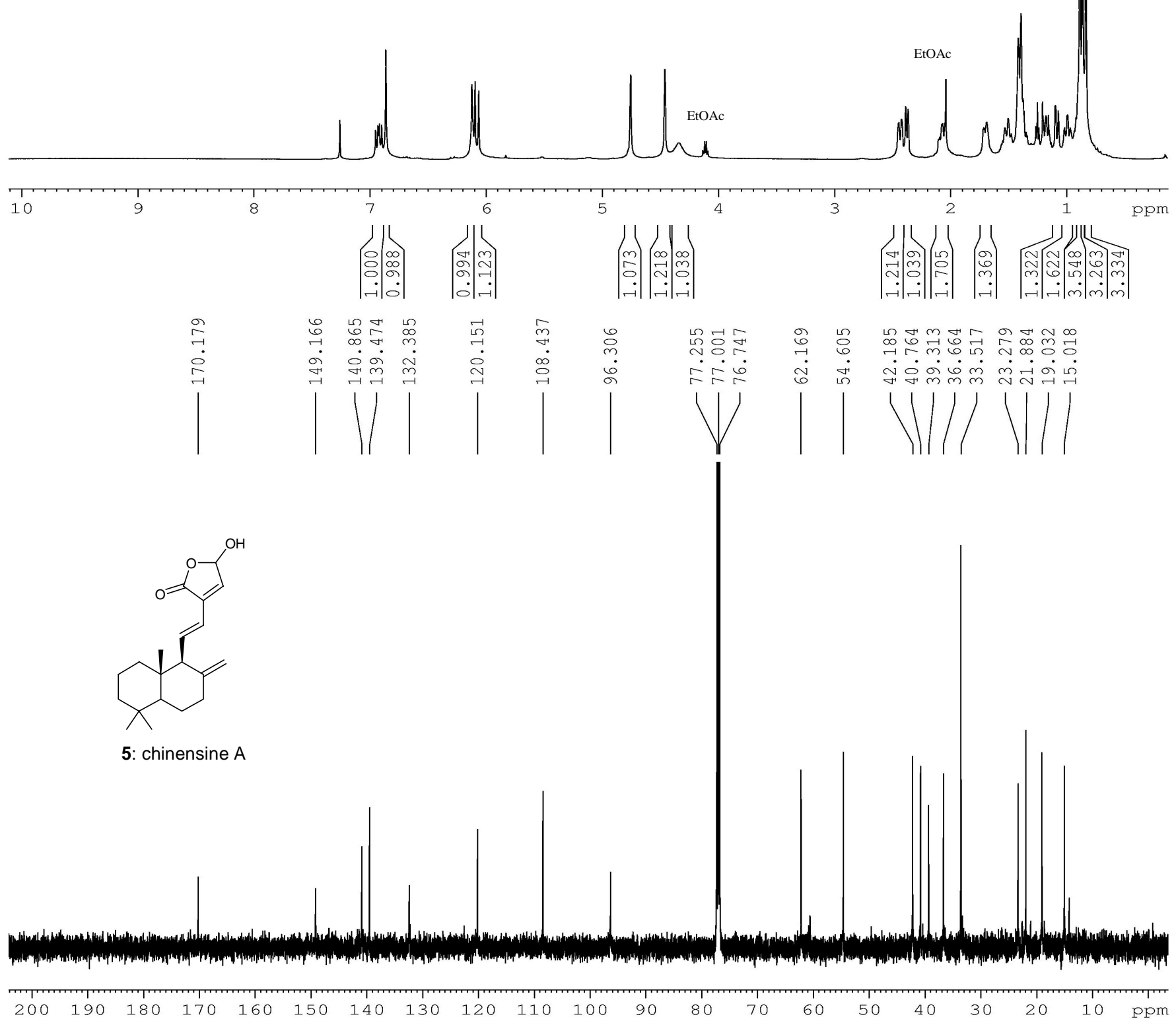




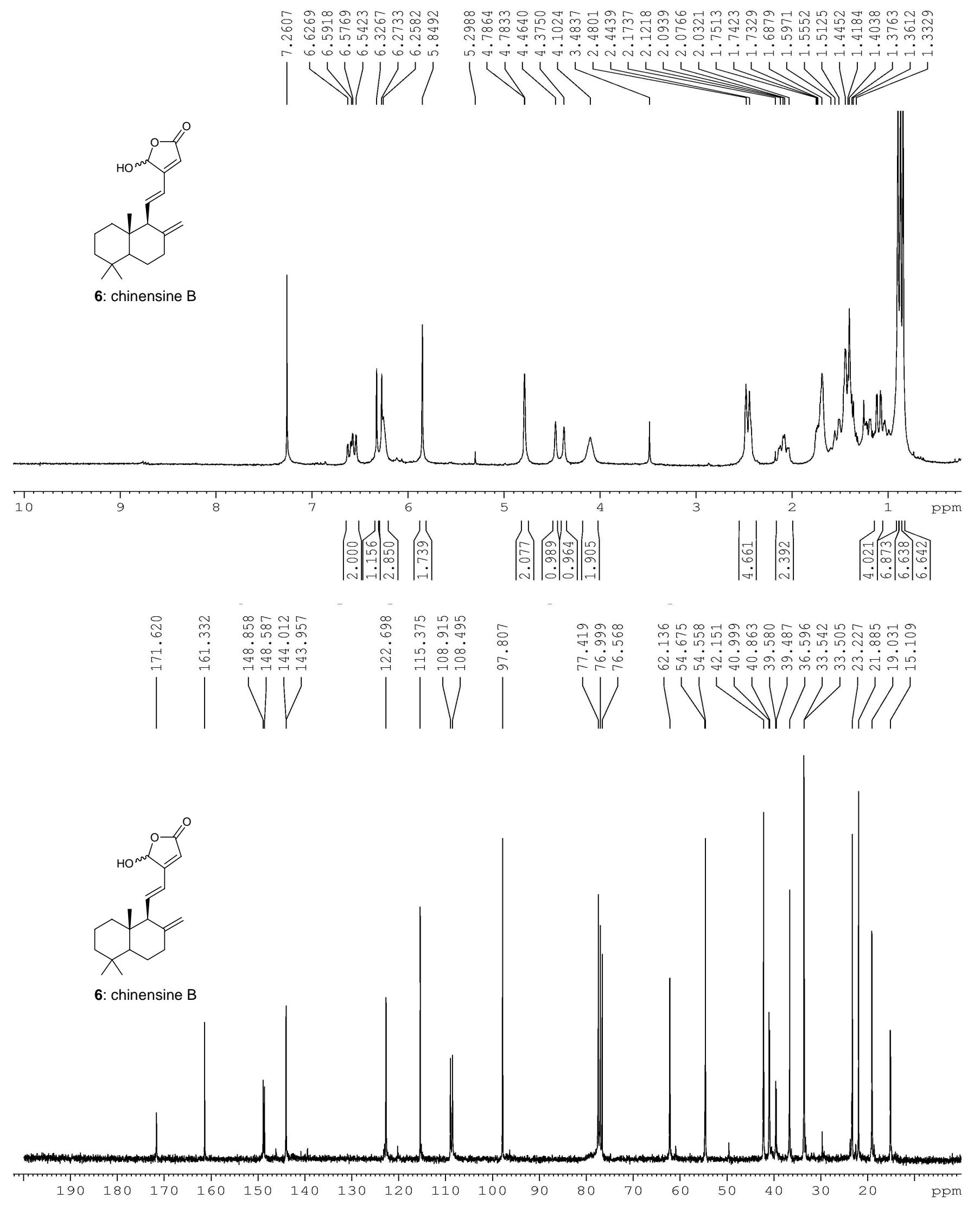




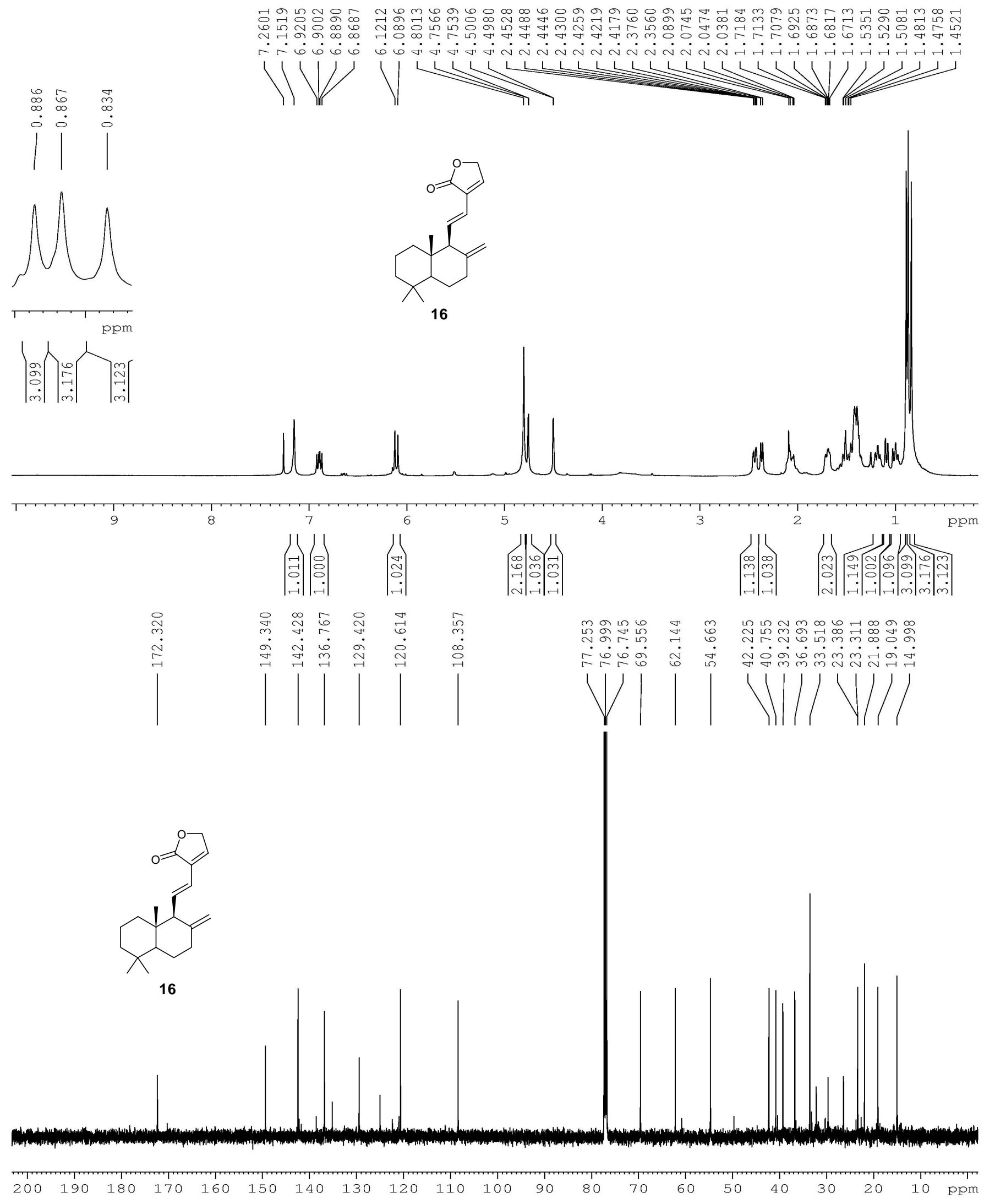




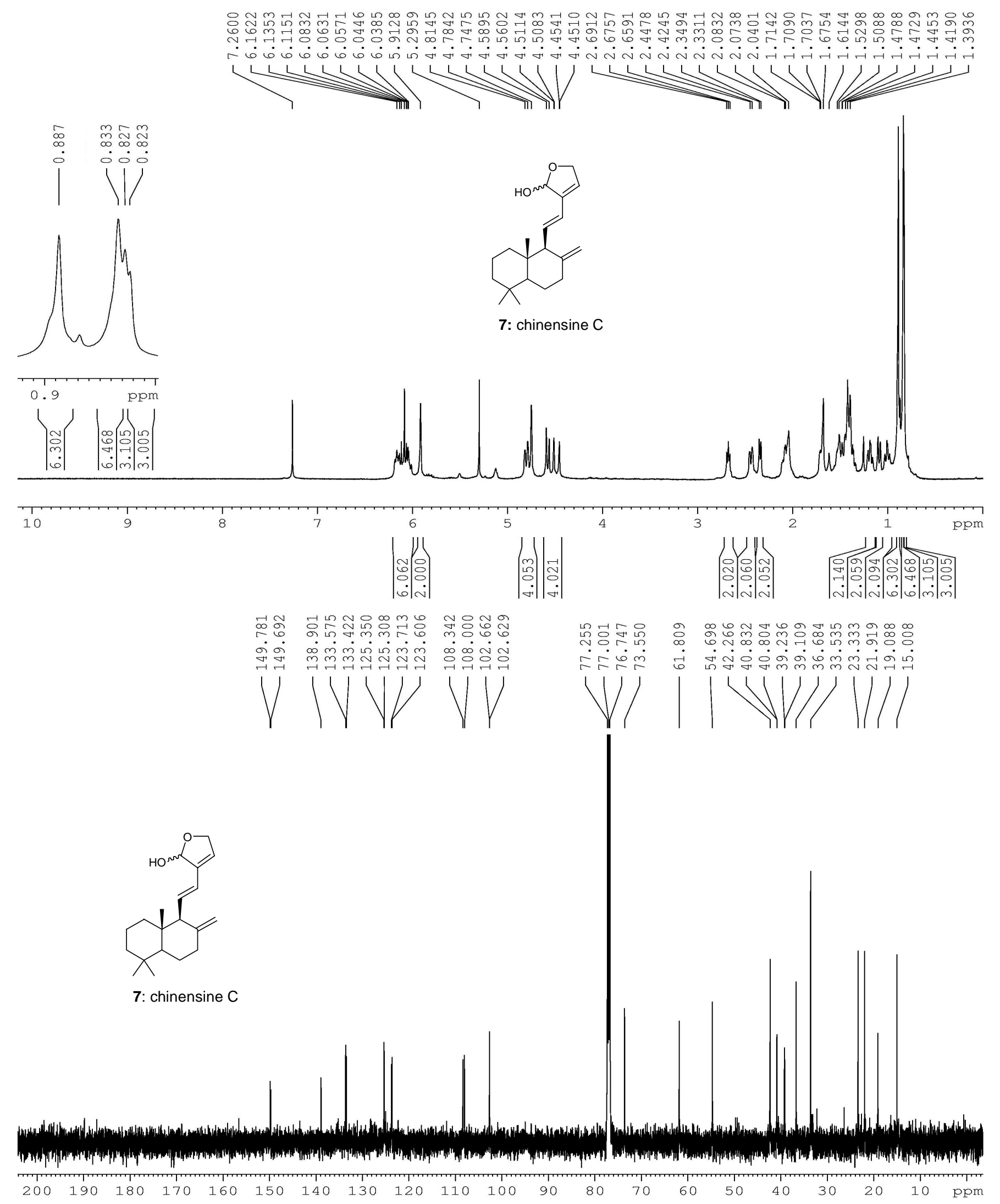




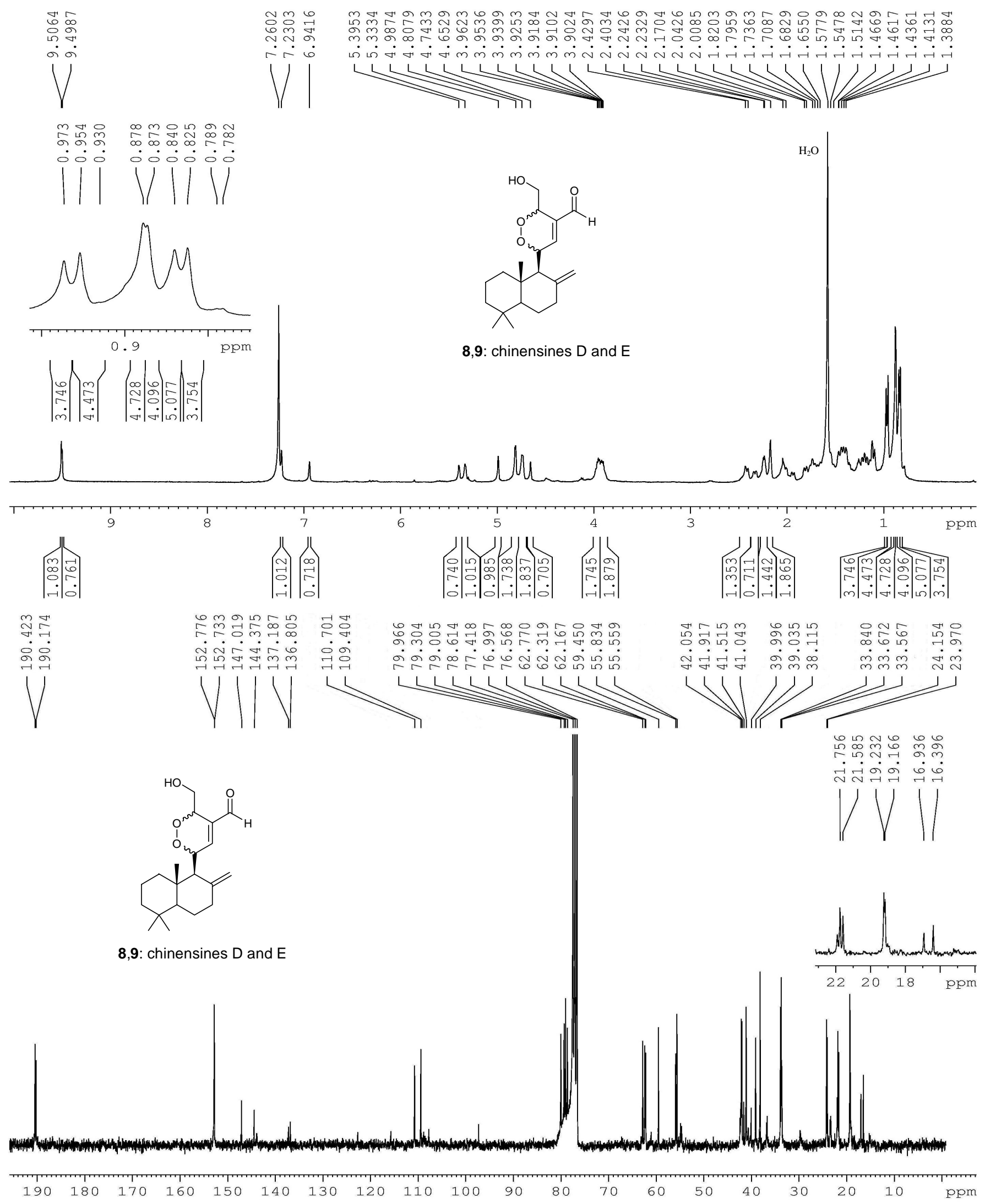

